\title{
The DEAR experiment-first results on kaonic hydrogen
}

\author{
J. Zmeskal ${ }^{\mathrm{a}, *}$, G. Beer ${ }^{\mathrm{b}}$, A.M. Bragadirean ${ }^{\mathrm{c}, \mathrm{d}}$, M. Cargnelli ${ }^{\mathrm{a}}$, \\ C. Curceanu (Petrascu) ${ }^{\text {c,d }}$, J.-P. Egger ${ }^{\text {e }}$, H. Fuhrmann ${ }^{a}$, \\ C. Guaraldo ${ }^{\mathrm{c}}$, M. Iliescu ${ }^{\mathrm{c}, \mathrm{d}}, \mathrm{T}$. Ishiwatari ${ }^{\mathrm{a}}, \mathrm{K}$. Itahashi ${ }^{\mathrm{f}}$, \\ M. Iwasaki ${ }^{\mathrm{f}}$, P. Kienle ${ }^{\text {a }}$, B. Lauss ${ }^{\mathrm{g}}$, V. Lucherini ${ }^{\mathrm{c}}$, L. Ludhova ${ }^{\text {h }}$, \\ J. Marton ${ }^{\text {a }}$, F. Mulhauser ${ }^{\mathrm{h}}$, T. Ponta ${ }^{\mathrm{d}}$, L.A. Schaller ${ }^{\mathrm{h}}$, R. Seki ${ }^{\mathrm{i}, \mathrm{j}}$, \\ D.L. Sirghi ${ }^{c, d}$, F. Sirghi ${ }^{c}$ \\ ${ }^{a}$ Stefan Meyer Insitut fuer subatomare Physik, Austrian Academy of Sciences, Boltzmanngasse 3, \\ A-1090 Vienna, Austria \\ b University of Victoria, Department of Physics and Astronomy, P.O. Box 3055 Victoria, BC V8W 3P6, Canada \\ ${ }^{\mathrm{c}}$ INFN, Laboratori Nazionali di Frascati, C.P. 13, Via E. Fermi 40, I-00044 Frascati, Italy \\ $\mathrm{d}$ Institute of Physics and Nuclear Engineering "Horia Hulubei", P.O. Box R-76900 Magurele, \\ Bucharest, Romania \\ e University de Neuchatel, Institute de Physique, Université de Neuchâtel,1 rue A.-L. Breguet, \\ CH-2000 Neuchâtel, Switzerland \\ ${ }^{\mathrm{f}}$ Institute of Physical and Chemical Research (RIKEN), 2-1 Hirosawa, Wako, Saitama 351-01, Japan \\ g University of California, Department of Physics, Le Conte Hall 366, Berkeley, CA 94720, USA \\ ${ }^{\mathrm{h}}{ }^{\mathrm{L}}$ Université de Fribourg, Institute de Physique, Bd. de Perolles, CH-1700 Fribourg, Switzerland \\ ${ }^{\mathrm{i}}$ W.K. Kellog Radiation Laboratory, California Institute of Technology, Pasadena, CA 91125, USA \\ j Department of Physics and Astrophysics, California State University, Northridge, CA 91330, USA \\ Received 17 December 2004; accepted 8 February 2005 \\ Available online 3 March 2005

\begin{abstract}
\footnotetext{
* Corresponding author.

E-mail address: johann.zmeskal@oeaw.ac.at (J. Zmeskal).
} \\ The goal of the DEAR (DA $\Phi$ NE exotic atom research) experiment is the precise determination \\ of the isospin dependent antikaon-nucleon scattering lengths. The experiment accurately measures
}


the $K \alpha$ line shift and broadening, due to the strong interaction, in kaonic hydrogen and, for the first time, in kaonic deuterium. A precision measurement of kaonic hydrogen tests chiral symmetry breaking in systems with strangeness. An initial analysis of the DEAR experiment yields a shift $\varepsilon_{1 s}=-195 \pm 45 \mathrm{eV}$ and a width $\Gamma_{1 s}=250 \pm 125 \mathrm{eV}$, which is more precise than the previous kaonic X-ray experiment $\mathrm{KpX}$ at KEK, and allows for the first time to disentangle the full pattern of the kaonic hydrogen $K$-series line $K \alpha, K \beta$ and $K \gamma$.

\section{Introduction}

This research deals with the characteristic properties of the strong interaction of antikaons $(K)$ with nucleons $(p)$. These hadrons consist of light quarks $(u ; d ; s)$ and have masses which are considerably larger than their current quark masses. This extraordinary phenomenon is proposed to originate from spontaneous breaking of chiral symmetry of massless quarks in strong interaction physics [1]. It results in a ground state-the vacuum state - with a finite expectation value of quark-antiquark pairs, the chiral quark condensate $[2,3]$. The hadrons are considered as quasiparticle excitations of this chiral condensate. The lowest excitation modes of the condensate, the so-called Nambu-Goldstone bosons [1], are identified as mesons containing light quarks. Their $s$ wave interaction with nucleons is determined by the pion and kaon decay constants $f_{\pi}$ and $f_{K}$, respectively, the squares of which are also the order parameters of chiral symmetry breaking [4,5] and are directly connected to the magnitude of the quark condensate through the Gell-Mann-Oaks-Renner relation [5]. By comparison of the $s$-wave pion-nucleon and pion-nucleus interaction it was found recently that chiral symmetry is partially restored in a nuclear medium in agreement with the expectation of chiral dynamics [6,7].

In this work, the chiral symmetry breaking scenario in the strangeness sector is investigated by studying the $K^{-} p s$-wave interaction at threshold by measuring precisely the energies and widths of the X-ray transitions in kaonic hydrogen. In a previous experiment Iwasaki et al. [8] and Ito et al. [9] found that the strong interaction shift of the $1 s$ state in kaonic hydrogen is repulsive, in agreement with scattering data and in disagreement with earlier results, thus resolving the so-called "kaonic hydrogen puzzle" (wrong sign of shifts from previous experiments). The origin of this repulsive strong interaction at threshold can be traced back to the presence of the $\Lambda(1405)$ resonance which leads on one hand to a repulsive $K^{-} p$ scattering length and on the other to the possible existence of strongly bound kaonic states in light nuclei [10]. The most basic state, $p p K^{-}$, can be considered as a strongly bound "kaonic hydrogen" molecule. Very recently, searches for such exotic kaonic cluster states were performed and evidence has been reported for a $p p n K^{-}$state with a binding energy of about $170 \mathrm{MeV}$ and a width of about $20 \mathrm{MeV}$ [11].

In view of these developments and the fundamental importance of the $s$-wave $K N$ scattering length for chiral dynamics with strangeness, it is of utmost importance to confirm the finding of the $\mathrm{KpX}$ experiment [8] and determine more precisely the strong interaction shift and width of kaonic hydrogen. 


\section{The DEAR experimental setup}

The DEAR experiment makes use of low momentum negative kaons, produced by the decay of the $\Phi$ meson at DA $\Phi$ NE. The kaons leave the interaction point through the DEAR beam pipe made out of thin-walled aluminum with carbon fiber reinforcement. They are degraded in energy to a few $\mathrm{MeV}$ and enter a gaseous hydrogen target placed about $10 \mathrm{~cm}$ above the beam pipe where they are stopped. The cylindrical cryogenic target cell has a diameter of $12.5 \mathrm{~cm}$ and a height of $14 \mathrm{~cm}$. Special care was taken to avoid materials with fluorescence X-ray radiation in the energy region of interest. Therefore, a light weight target was chosen, made only of aluminum (top-plate and entrance-ring), Kapton (side wall and entrance window) and a support structure of fiberglass reinforced epoxy.

The hydrogen gas target cell, containing ultra pure hydrogen cleaned through a palladium diffusion device, typically works at a gas pressure of 2 bar and a temperature of $23 \mathrm{~K}$, stabilized to better than $0.1 \mathrm{~K}$. With this setting, a gas density of $2.2 \mathrm{mg} / \mathrm{cm}^{3}(3.1 \%$ of liquid hydrogen density-LHD) is achieved, corresponding to a gas pressure at room temperature of about 30 bar. The insulation vacuum is maintained to better than $10^{-6} \mathrm{mbar}$ using a wide-range turbo molecular pump (see Fig. 1).

The detector contains 16 CCD chips (Marconi Applied Technologies, CCD55-30) with a total area of $116 \mathrm{~cm}^{2}$, which were placed around the cryogenic target cell. Each chip has $1242 \times 1152$ pixels with a pixel size of $22.5 \times 22.5 \mu \mathrm{m}^{2}$ and a depletion depth of about $30 \mu \mathrm{m}$. The working temperature of the CCD chips was stabilized at $165 \mathrm{~K}$ to achieve an energy resolution of $150 \mathrm{eV}$ at $6 \mathrm{keV}$ using a read out time of 90 seconds.

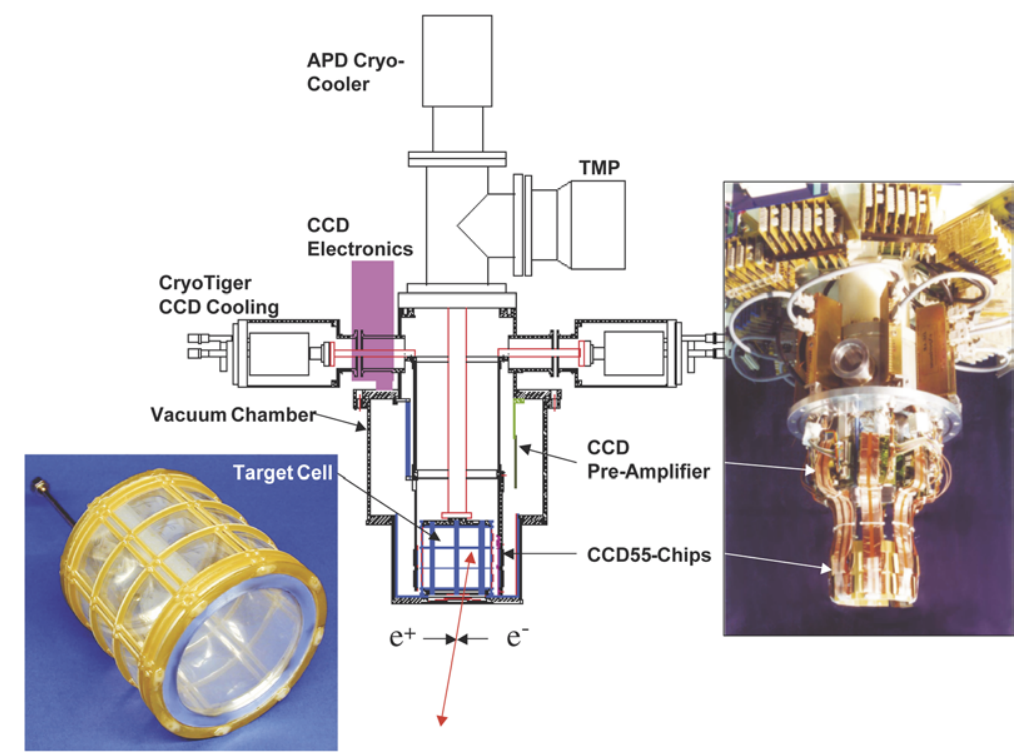

Fig. 1. The DEAR setup: a cryogenic lightweight target cell surrounded by a CCD-detector inside a common vacuum housing. 
The production rate of charged kaons was measured by two plastic scintillators which detected kaons emitted back-to-back [12]. The kaon's initial energy of $16 \mathrm{MeV}$ was reduced by plastic degraders to $3 \mathrm{MeV}$ which allows them to stop within a few $\mathrm{cm}$ in the hydrogen gas.

Sophisticated shielding of the DEAR target and detector was developed through different test runs at DA $\Phi$ NE. Finally, a graded shielding structure was used, starting with lead, followed by a copper and an aluminum layer, with an inner layer of polycarbonate. With this setup the bremsstrahlung background could be reduced drastically, as proven using nitrogen as target gas.

\section{Kaonic nitrogen}

For a measurement of kaonic nitrogen transitions [13], nitrogen gas at $120 \mathrm{~K}$ and $1.5 \mathrm{bar}$ was used instead of hydrogen. This measurement was done immediately before the hydrogen run and was also used as a background measurement for the kaonic hydrogen data. Data corresponding to an integrated luminosity of $32 \mathrm{pb}^{-1}\left(45.6 \times 10^{6} \mathrm{~K}^{-}\right)$were taken for nitrogen.

In addition, the kaonic nitrogen measurement is essential to tune the machine and to optimize the DEAR apparatus for the kaonic hydrogen experiment. Improvements of the detector shielding (signal to background) as well as an optimization of the kaon stopping distribution in the gaseous target cell could be measured directly with kaonic nitrogen $\mathrm{X}$-rays within a few days.

\section{Kaonic hydrogen first results}

The experimental challenge of DEAR is the extraction of a small signal in the presence of a large low-energy X-ray background mainly from electron gamma showers resulting from lost electrons and positrons due to either Touschek scattering or beam interactions with residual gas. The careful optimization of the shielding of our experimental setup and the improvements in the beam optics achieved by the machine crew made the goal of performing the first measurement of kaonic hydrogen at DA $\Phi$ NE, possible.

Although all material used for the target cell and the mounting device for the CCDs was carefully checked, it was not possible to completely avoid iron impurities. The problem with iron impurities is that the iron fluorescence line overlaps partly the kaonic hydrogen $K \alpha$ line. Based on the two background measurements-with kaonic nitrogen as target gas and the other with hydrogen, but with the beams separated, (meaning no collisions and therefore no kaons produced) - it was possible to determine the iron content and to subtract the iron fluorescence line from the kaonic $K \alpha$ line.

The linearity as well as the energy stability of the CCD detector was measured in situ using the titanium and zirconium lines (these foils were placed on top of the target cell). The CCD detector system was extremely stable during beam time. Stability was checked by fixing the energy position of the $\mathrm{Ti}$ and $\mathrm{Zr}$ lines and measuring the position of the $\mathrm{Ca}$ line which was fitted to an accuracy of better than $1 \mathrm{eV}$. In addition, the Ti $K \alpha$ line width 


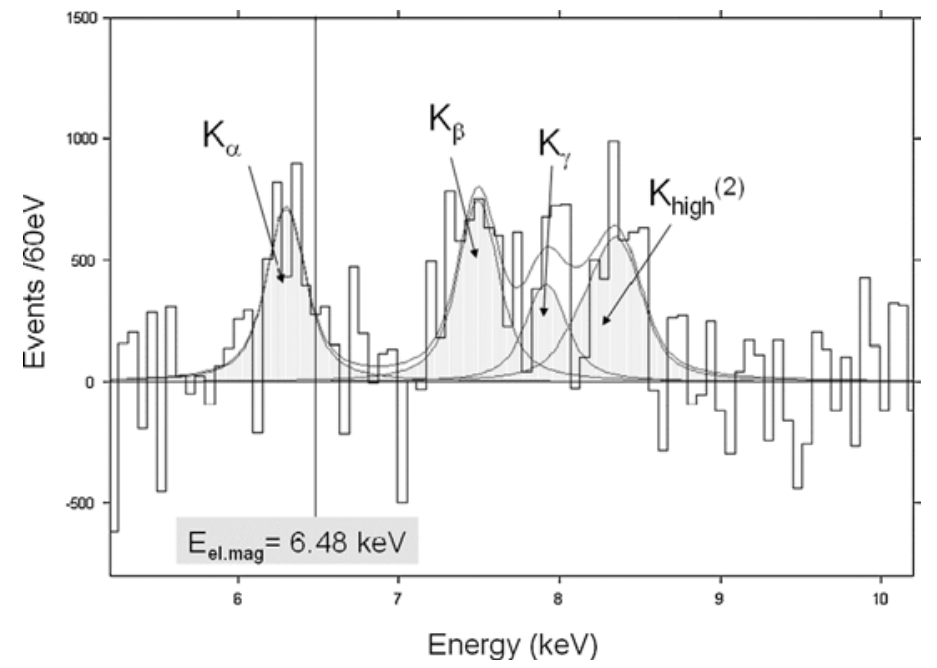

Fig. 2. Background subtracted kaonic hydrogen spectrum for an integrated luminosity of $60 \mathrm{pb}^{-1}$; the kaonic hydrogen $K \alpha$-, $K \beta$ - and $K \gamma$-line are fitted.

for the sum of all CCDs (finally only 14 of 16 CCDs were used) was better than $150 \mathrm{eV}$ for the whole beam time.

Two completely independent analyses starting from the raw data set were performed. The main differences were the treatment of the continuous background and the determination of the fluorescence X-ray lines.

Fig. 2 shows the resulting kaonic hydrogen spectrum with the continuous background as well as the fluorescence X-ray lines subtracted. The $K \alpha$ line together with a bump corresponding to the $K$ complex are clearly visible. Both analysis methods gave a compatible result:

$$
\begin{aligned}
& \varepsilon_{1 s}=-195 \pm 45 \mathrm{eV}, \\
& \Gamma_{1 s}=250 \pm 125 \mathrm{eV} .
\end{aligned}
$$

The result is consistent with the values obtained in the KpX experiment at KEK [8] and confirms the repulsive character of the $K^{-} p$ interaction at threshold. For the first time the $K$-series lines $K \alpha, K \beta$ and $K \gamma$ of kaonic hydrogen are clearly identified. Furthermore, the DEAR errors are $2-3$ times smaller than in the KpX experiment.

\section{Summary}

In summary, the analysis of the first measurement of kaonic hydrogen at DAФNE already leads to an improved accuracy in the determination of the shift and width of the ground state of kaonic hydrogen and confirms the repulsive contribution of the strong interaction (as well as the KpX experiment at KEK, Japan). Theoretical predictions based on chiral perturbation theory and a recent quantum field theoretical approach can now be confronted with our new result $[14,15]$. 


\section{Acknowledgements}

The DAФNE group is warmly acknowledged for the excellent cooperation and teamwork. Part of the work was supported by a "Transnational Access to Research Infrastructure" (TARI), Contract No. HPRI-CT-1999-00088.

\section{References}

[1] Y. Nambu, G. Jona-Lasinio, Phys. Rev. 122 (1961) 345.

[2] T. Hatsuda, T. Kunihiro, Phys. Rep. 247 (1994) 221.

[3] U. Vogl, W. Weise, Prog. Part. Nucl. Phys. 27 (1991) 195, and references therein.

[4] M. Gell-Mann, M. Levy, Nuovo Cimento 16 (1960) 706.

[5] M. Gell-Mann, R.J. Oakes, B. Renner, Phys. Rev. 175 (1968) 2195.

[6] K. Suzuki, et al., Phys. Rev. Lett. 92 (2004) 072302.

[7] P. Kienle, T. Yamazaki, Prog. Part. Nucl. Phys. 52 (2004) 85.

[8] M. Iwasaki, et al., Phys. Rev. Lett. 78 (1997) 3067.

[9] T.M. Ito, et al., Phys. Rev. C 58 (1998) 2366.

[10] Y. Akaishi, T. Yamazaki, Phys. Rev. C 65 (2002) 44005.

[11] M. Iwasaki, in preparation.

[12] V. Lucherini, et al., Nucl. Instrum. Methods A 496 (2003) 315.

[13] T. Ishiwatari, et al., Phys. Lett. B, in press.

[14] J. Gasser, in: Mini Proceedings CD 2003, hep-ph/0311212.

[15] A. Ivanov, et al., nucl-th/0310081, Eur. Phys. J. A submitted for publication. 